\title{
Atomic Scale Structural Analysis of Sn-Si Quantum Dots
}

\author{
Y. Lei, ${ }^{*}$ P. Möck, ${ }^{*}$ T. Topuria, ${ }^{*}$ N. D. Browning, ${ }^{*}$ R. Ragan, ${ }^{* *}$ H. A. Atwater ${ }^{* *}$ \\ * Dept. of Physics, University of Illinois at Chicago, 845 W. Taylor St., Chicago, IL 60607, USA \\ ** Thomas J. Watson Laboratory of Applied Physics, California Institute of Technology, MS 128-95, \\ Pasadena, CA 91125, USA
}

The interest in group IV semiconductor quantum dots containing $\mathrm{Sn}$ in a $\mathrm{Si}$ matrix is motivated by their potential applications for band-structure engineered direct-band-gap element semiconductors for optoelectronics and thermophotovoltaics [1]. Recently, ultrathin pseudomorphic $\mathrm{Sn}_{0.1} \mathrm{Si}_{0.9}$ layers embedded in Si matrix were grown by temperature-modulated molecular beam epitaxy, and postgrowth thermal annealing resulted in Sn-rich quantum dots (QDs) via phase separation [2]. Since the crystallographic phase and chemical composition within the dots are crucial for the properties of QD devices, it is necessary to characterize the QD materials on the atomic scale. Here we report a study of QDs that formed from a $\mathrm{Sn}_{0.1} \mathrm{Si}_{0.9}$ multilayer system in a $\mathrm{Si}(001)$ crystal [2] by a combination of Z-contrast imaging in a scanning transmission electron microscope (STEM) and electron energy loss spectroscopy (EELS).

The experimental analysis was performed on a $200 \mathrm{kV}$ Schottky field emission JEOL JEM 2010F STEM/TEM. This microscope is capable of obtaining Z-contrast images, which are essentially incoherent, that can be used for an intuitive interpretation of the atomic structure of Sn-Si samples less than $\sim 100 \mathrm{~nm}$ thick. The EELS technique allows fluctuations in chemical composition to be quantified on the sub-nm scale and assessments of the local electronic structure made. When combined with Z-contrast imaging, it is possible to obtain spectra with atomic spatial resolution [3]. One advantage of the Z-contrast method is that the analyses are not influenced by the strain field which surrounds QDs.

A [110] cross-sectional TEM specimen of the $\mathrm{Sn}_{0.1} \mathrm{Si}_{0.9} / \mathrm{Si}$ multilayer was prepared by standard procedures. Typical diameters of the QDs were ranging from 7 to $10 \mathrm{~nm}$. Figure 1 shows a Zcontrast image of a QD that consists of $\alpha$-Sn (i.e. semiconducting $\mathrm{Sn}$, diamond structure, $\left.\mathrm{F} 4_{1} / \mathrm{d} \overline{3} 2 / \mathrm{m}\right)$. The QDs appear to possess a more or less developed octahedral habit that also contain $\{110\}$ and $\{100\}$ facets. The presence of all three kinds of facets is consistent with the equilibrium shape of the voids in $\mathrm{Si}$ [4]. Figure $2 \mathrm{a}$ and $2 \mathrm{~b}$ show Z-contrast images of two QDs that consist of $\beta$ Sn (i.e. metallic Sn, $\mathrm{I}_{1} / \mathrm{a} 2 / \mathrm{m} 2 /$ d) and possess different orientation relationships with the Si matrix (as can also be inferred from the inserted Fourier transforms). Using sub-nm resolution EELS spectra, a low loss peak at $14 \mathrm{eV}$ was observed for both of these " $\beta$-Sn" QDs.

Figure 3a shows a Z-contrast image of a novel type of QD with periodic compositional modulation formed inside the dot. The compositional modulation was parallel to the [001] growth direction, and the whole entity seems to possess the diamond structure. The quantitative analysis of Sn M-edge spectra confirmed a modulation in the Sn content as shown in Fig. 3b. Taking into account the extremely low solid solubility of $\mathrm{Sn}$ in $\mathrm{Si}(<0.1 \%)$ and the large mismatch between $\alpha-\mathrm{Sn}$ and $\mathrm{Si}$ $(19.5 \%)$, this phenomenon might be caused by a strain relaxation process similar to that observed in III-V QDs [5]. Hypotheses on the formation mechanism of the three observed types of QDs and details of the observed orientation relationships will be discussed elsewhere. 


\section{References}

[1] R. Soref, and A. C. H. Perry, J. Appl. Phys. 69 (1991) 539.

[2] K. S. Min, and H. A. Atwater, Appl. Phys. Lett. 72 (1998) 1884.

[3] N. D. Browning et al., Nature 366 (1993) 143.

[4] D. J. Eaglesham et al., Phys. Rev. Lett. 70 (1993) 1643.

[5] P. Möck et al., Appl. Phys. Lett. 79 (2001) 946.

(1)

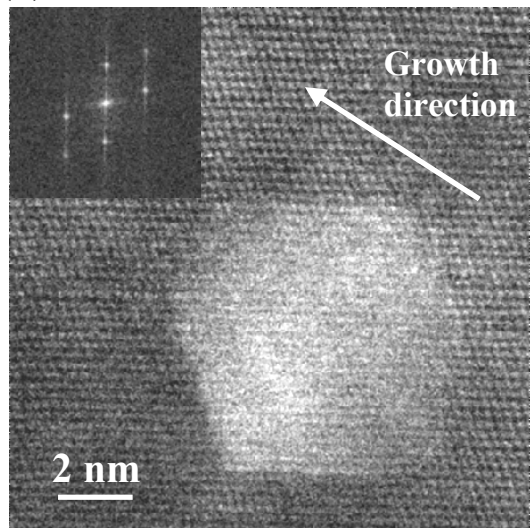

Fig. 1. $\mathrm{Z}$ contrast image of a quantum dot consisting of $\alpha-\mathrm{Sn}$ (growth direction [001] as indicated, same for other images)

\section{(2a)}

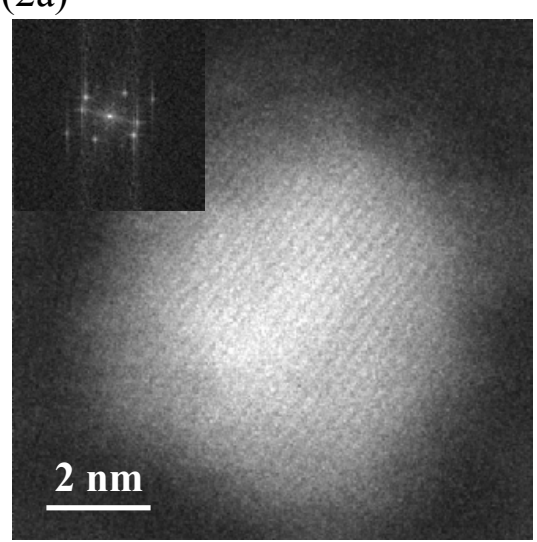

(2b)

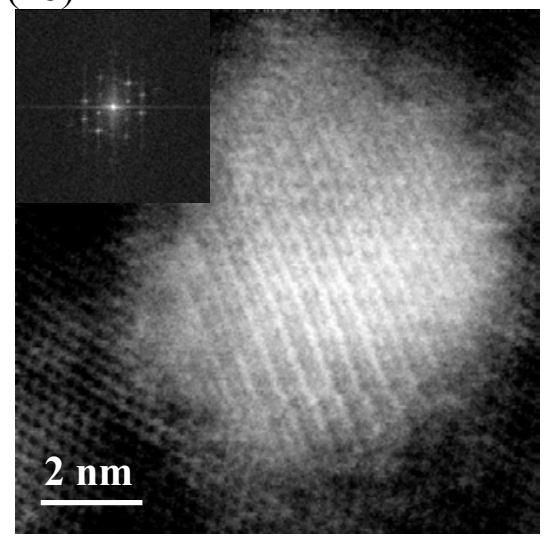

Fig. 2. $\mathrm{Z}$ contrast images of two quantum dots that consist of $\beta$-Sn and possess different orientation relationships with the Si matrix

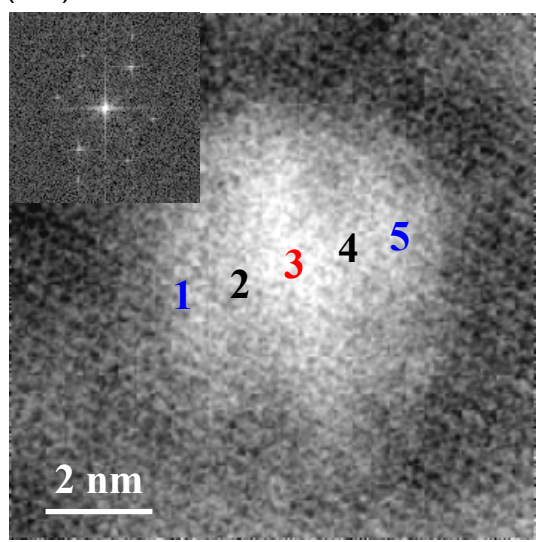

\section{(3a)}

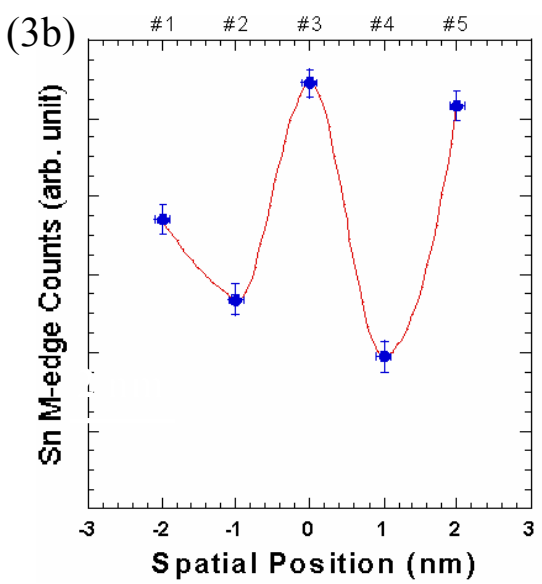

Fig. 3. (a) Z-contrast image of a novel type of quantum dot with internal periodic compositional modulation;

(b) Sn M-edge counts variation at the spatial positions indicated in (a)

* Electronic mail: ylei1@uic.edu 